\title{
La orientación como agente de resiliencia en estudiantes universitarios
}

\author{
Guidance as a resilience agent in university students
}

\author{
- DELEANA PAOLA RINCÓN FUENMAYOR \\ deleana@gmail.com \\ Código ORCID: 0000-0001-8438-0044 \\ Universidad del Zulia, Venezuela
}

Artículo recibido en abril 2020 / Arbitrado en mayo 2020 / Publicado en julio 2020

Resumen Se plantea determinar la resiliencia por medio de los factores resilientes presentes en los estudiantes de Orientación para el egreso. Enfocando la resiliencia desde la perspectiva de Henderson Grotberg (2003).Considerándose una investigación descriptiva, midiéndose los factores de resiliencia presentes en 37 estudiantes; ubicados entre el $7 \mathrm{mo}$ y 9 no semestre de Ingeniería civil y petróleo. Aplicando un instrumento elaborado por Rosalva Teyes en su investigación: "Resiliencia y Rendimiento académico en estudiantes universitarios" (RESI-TEYES-LUZ) tipo Likert, con un índice de confidencialidad de 0,743 y un tratamiento estadístico descriptivo de censo poblacional Como conclusión se tiene que los estudiantes poseen resiliencia con tendencia alta a mediana, considerando debilidad en los factores internos Yo Soy, en las relaciones interpersonales y estabilidad emocional, así como en los factores externos Yo Tengo, en cuanto al entorno social y familia estable.

Palabras clave: Resiliencia; estudiantes; adversidades; fortaleza

\begin{abstract}
It is proposed to determine resilience through the resilience factors present in the orientation students for graduation. Approaching resilience from the perspective of Henderson Grotberg, (2003). Considering a descriptive investigation, measuring the resilience factors present in 37 students; from the 7th and 9th semester of civil and petroleum engineering schools. Applying an instrument prepared by Rosalva Teyes in her research: "Resilience and Academic Performance in University Students" (RESI-TEYES-LUZ) type Likert, with a confidentiality index of 0.743 ; and descriptive statistical treatment of population census. As a conclusion, students have resilience with a high to medium tendency, considering weakness in the internal factors I am, in interpersonal relationships and emotional stability, as well as in external factors I have, in regarding the social environment and stable family.
\end{abstract}

Keywords: $\quad$ Resilience; students; adversities; strength 


\section{INTRODUCCIÓN}

Desde hace varios años, a nivel mundial, ya se han venido desarrollando políticas educativas universitarias que permiten no solo formar desde el punto de vista técnico o de conocimiento puro, sino también desde una dimensión holística donde cada individuo aprenda a desarrollar un mayor compromiso consigo mismo y crecimiento personal, así como también contar con la disposición de trabajar inmerso en una sociedad, aprendiendo a adaptarse a nuevos escenarios y ambientes.

El proceso de transición universitaria es de gran trascendencia e impacto en los estudiantes, especialmente en momentos de crisis, las cuales se presentan a lo largo de la vida en cualquiera de sus etapas de desarrollo del ciclo vital, influyendo en su manera de pensar, sentir y accionar. Es por ello que se requiere investigar el nivel de resiliencia que manejan los estudiantes a través de los factores resilientes en los estudiantes de Orientación para el egreso de la Facultad de Ingeniería de la Universidad del Zulia, puesto que están en la etapa de la vida que caracteriza una serie de cambios a nivel psicológico, cognoscitiva y comportamental adentrando a otra etapa que los podría ubicar en una posición vulnerable ante los escenarios de adversidad que atraviesa el país.

Se entiende entonces que los factores resiliente están presentes en las personas, pero estos no siempre son evidentes, es necesario que logren identificar cuáles son sus recursos internos y externos de manera que puedan enfrentar la adversidad con una actitud positiva, esperanzadora y comprender que a pesar de no poder cambiar la realidad, tienen la opción de cambiar la actitud de afrontamiento.
Para esta investigación se toma en cuenta el modelo de servicios en orientación, en el contexto educativo ya que busca dar respuesta, de manera sistemática, y apoyo a las determinadas circunstancias planteadas por el sistema humano a abordar. La orientación constituye una preparación para todas las etapas del desarrollo evolutivo del estudiante así como también de la preparación del mismo en su desempeño profesional, considerándose como una orientación académico profesional

El orientador como promotor e interventor con el fin de participar activa mente en el proceso de transformaciones de los grupos humanos involucrados en esta investigación. Trazando como objetivo general: Determinar la resiliencia por medio de los factores resilientes en los estudiantes de Orientación para el egreso de la Facultad de Ingeniería en la Universidad del Zulia, el cual se responderá a través de los objetivos específicos: identificar los factores internos de la resiliencia relacionados con el Yo Puedo, caracterizar los factores internos de la resiliencia relacionados con el Yo Soy, describir los factores externos de la resiliencia relacionados con el Yo Tengo.

La investigación está justificada desde el punto de vista teórico debido a que la temática se encuentra vigente y necesaria para estos tiempos de crisis que vive la humanidad, especialmente el estudiante venezolano. Metodológicamente conlleva a un aporte científico por la aplicación de un instrumento que permite explorar los factores internos $y$ externos de resiliencia que poseen los estudiantes para controlar los escenarios de adversidad y abrir otras investigaciones que se adentren y desarrollen puntos específicos de 
este tema. De acuerdo a los resultados obtenidos se desprende un senvicio de orientación que permita potenciar la resiliencia en los estudiantes, así como desde el aspecto científico permitirá dar vías de solución y al desarrollo de los factores resilientes en los estudiantes.

\section{Algunas nociones claves}

Cabe destacar que el planteamiento teórico utilizado para esta investigación se corresponde al descrito por Henderson Grotberg (2003), el cual caracteriza la presencia de factores internos y externos que circundan al ser humano a lo largo de su ciclo de vida. Entendiendo entonces que el mismo responderá a los objetivos planteados por la investigadora.

Etimológicamente, el término resiliencia proviene del latín resilio, que significa volver atrás, rebotar o resurgir (Kotliarenco, Cáceres y Fontecilla, 1997).Utilizado tradicionalmente en la investigación física, se refiere a la capacidad de un material para recobrar su forma después de haber estado sometido a altas presiones.

Más tarde, fue adoptado por la Psicología, con un significado muy cercano al original referido a rebotar de una experiencia difícil, como si uno fuera una bola o un resorte (Luthar, Sawyer y Brown., 2006). De esta manera, se comenzó a considerar la resiliencia como un aspecto consistente en una buena adaptación a la adversidad, a un trauma, una tragedia, amenaza, o a fuentes de tensión significativas, como problemas familiares $\mathrm{o}$ de relaciones personales, problemas de salud o situaciones estresantes del trabajo o la educación. (Rutter, 1987)
Desde 2011, se encuentra incluido en el Diccionario de la Real Academia Española cuyo significado es: "la capacidad humana de asumir con flexibilidad situaciones límite y sobreponerse a ellas" (s.n.). Como se señaló, la complejidad y evolución del término, da origen a diversas definiciones de resiliencia, que responden además, al enfoque adoptado.

\section{Factores de Resiliencia por Henderson Grotberg}

Los aportes iníciales sobre la resiliencia, generados por Rutter (1976), y Werner y Smith (1982); abordaban las características o factores que promueven la resiliencia, los cuales eran muchos, condujo a Henderson Grotberg a organizar estos factores en apoyos externos que promueve la resiliencia, la fuerza interior que se desarrolla a través del tiempo y por último los factores interpersonales, es decir, la capacidad de resolución de problemas. Para su mejor interpretación la autora agrupó estos factores en: YO TENGO, YO SOY, YO PUEDO.

Para Henderson Grotberg (2003) la resiliencia como la capacidad del ser humano para hacer frente a las adversidades de la vida, superarlas y ser transformados positivamente por ellas. Teniendo que el ser humano desarrolla las características resilientes a medida que va atravesando las diferentes etapas del ciclo evolutivo y con ello la verbalización y acción del Yo tengo, Yo soy, Yo puedo.

\section{Factores de la resiliencia}

YO TENGO: es producto de las relaciones de confianza y del apoyo formal e informal. (familia, colegio, la fe) 
a) Una o más personas dentro de mi grupo familiar en las que puedo confiar y me aman sin condicionamientos;

b) Una o más personas fuera de mi entorno familiar en las que puedo confiar plenamente.

c) Límites en mi comportamiento.

d) Personas que me incentivan a ser independiente.

e) Buenos modelos a imitar.

f) Acceso a la salud, a la educación y a los servicios de seguridad que necesito.

g) Una familia y un entorno social estable.

YO SOY: está relacionado con la fortaleza interna; atributos personales que fomentan la resiliencia.
a) Una persona que agrada a la mayoría de la gente.
b) Generalmente tranquilo y bien predispuesto.
c) Alguien que logra lo que se propone y que planea para el futuro.
d) Una persona que se respeta a sí misma y a los demás.

e) Alguien que siente empatía por los demás y se preocupa por ellos.

f) Responsable de sus propias acciones y acepto las consecuencias.

g) Seguro de sí mismo, optimista, confiado y con muchas esperanzas.

YO PUEDO: son las capacidades sociales e interpersonales, fundadas en lo personal y reafirmadas por la interacción social, en la resolución de conflictos.

a) Generar nuevas ideas o nuevos caminos para hacer las cosas.

b) Realizar una tarea hasta finalizar.

c) Encontrar el humor en la vida y utilizarlo para reducir tensiones.

d) Expresar sus pensamientos y sentimientos en su comunicación con los demás.

e) Resolver conflictos en diferentes ámbitos: académico, laboral, personal y social.

f) Controlar su comportamiento: sus sentimientos, sus impulsos, demostrar lo que siente.

g) Pedir ayuda cuando la necesita. 
La figura 1 muestra los Factores de Resiliencia por Henderson Grotberg (2005):

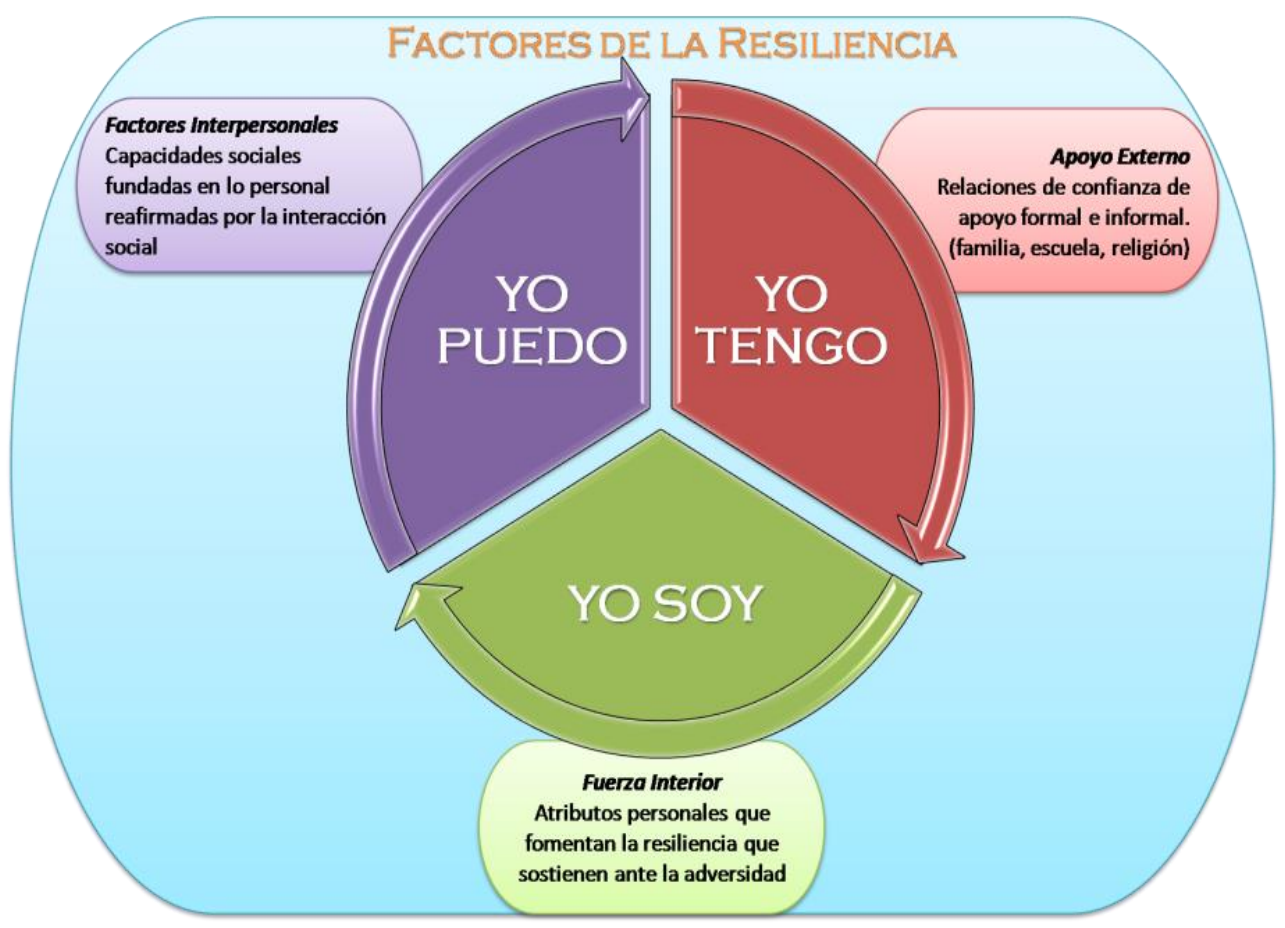

Figura 1. Factores de Resiliencia. (Fuente: Rincón, 2020)

MÉTODO

La metodología utilizada en el presente estudio conllevó al tipo de investigación descriptiva; ya que se hace la descripción, registro, análisis e interpretación de la variable resiliencia en los estudiantes de Orientación para el egreso en cuanto al diseño, es no experimental descriptivo transeccional ya que la variable resiliencia no es manipulada y los datos fueron recolectados en un momento y tiempo único.

En relación a la población de estudio se delimitó por los estudiantes de Orientación para el egreso de las escuelas de Petróleo y Civil cursantes de la asignatura Orientación para el egreso del Centro de Orientación y Promoción Integral CENOPI en la Facultad de Ingeniería de la Universidad del Zulia. Para este estudio fue considerado el total de la población, denominada Censo poblacional, conformada por
37 estudiantes: 20 de la escuela de Ingeniería en Petróleo y 17 de la escuela de Ingeniería Civil de la Universidad del Zulia. Adicionalmente se estimaron las técnicas e instrumentos que fueron empleados para la recolección de la información; $y$, finalmente, se especificó el procedimiento utilizado para analizar y discutir los datos obtenidos.

La información recabada se obtuvo a través de la encuesta como técnica de recolección de datos con la aplicación de un instrumento denominado Resi-teyes 2016, en formato tipo Lickert, desarrolla por la Dra. Rosalva Teyes en su Tesis doctoral titulada Resiliencia y rendimiento académico en estudiantes universitarios. Es oportuno destacar que se cumplieron con todos los lineamientos necesarios para su validez y confiabilidad, obteniendo una consistencia de 0,743 . 


\section{Baremo Ponderado para la interpretación de los estadísticos de la variable resiliencia}

Tabla 1. Baremo

\begin{tabular}{cc}
\hline CATEGORÍAS & PUNTUACIONES \\
\hline $1,00-1,80$ & Muy baja presencia \\
$1,81-2,60$ & Baja presencia \\
$2,61-340$ & Mediana Presencia \\
$3,41-4,20$ & Alta presencia \\
$4,21-5,00$ & Muy Alta presencia \\
\hline
\end{tabular}

\section{RESULTADOS Y DISCUSION}

Con respecto al análisis de los datos arrojados en esta investigación, se realizó un análisis estadístico por medio del programa de SPSS, el cual es el programa estadístico mayormente empleado en las investigaciones de las ciencias sociales. Una vez llevado a cabo dicho análisis estadístico, se realizó el proceso de interpretación de los resultados encontrados.

Se procedió a vaciar toda la información recopilada en tablas estadísticas utilizando como herramienta los programas estadísticos SPSS v27 y Microsoft Excel. Con los resultados obtenidos se pretende dar respuesta al objetivo general: determinar los factores resilientes en los estudiantes de Orientación para el egreso de la Facultad de Ingeniería en la Universidad del Zulia.

A continuación se muestran los resultados del primer objetivo que cita: Identificar los factores internos de la resiliencia relacionados con el Yo puedo en los estudiantes de Orientación para el egreso, que da respuesta a la dimensión factores internos Yo puedo con sus indicadores creatividad, persistencia, buen humor, asertividad, resolución de conflictos y pedir ayuda.
Los resultados expuestos para la dimensión factores internos desde la sub-dimensión Yo puedo reflejan que el $21.6 \%$, es decir 8 estudiantes considera que posee estas capacidades con muy alta presencia, el $45.9 \%$ lo que significa que 17 estudiantes manifestaron que la presencia de la dimensión es alta, el 27\% que representan a 10 encuestados expresan que medianamente presentan estas habilidades. Y, por último, el 5.4\% equivalente a 2 estudiantes reflejan que se logra con baja presencia este pilar.

Bajo la mirada de Henderson Grotberg puede interpretarse que los estudiantes de Orientación para el egreso en la sub-dimensión Yo puedo como factor interno de resiliencia con un tendencia alta - muy alta poseen desarrollada las cualidades que le permiten generar ideas y alternativas para hacer las cosas con humor reduciendo tensiones, establecer vínculos y comunicación con los demás controlando sus comportamientos sabiendo expresar lo que sienten y pedir ayuda si es necesario. Poseen las capacidades necesarias para enfrentar la adversidad. 
A continuación se muestran los resultados del segundo objetivo que cita Caracterizar los factores internos de la resiliencia relacionados con e/Yo soy en los estudiantes de Orientación para el egreso, que da respuesta a la dimensión factores internos Yo soy con sus indicadores relaciones interpersonales, estabilidad emocional, empatía, fijación de metas, respeto y optimismo.

Los resultados expuestos con base a la dimensión factores internos desde el factor Yo soy, por su parte reflejan que el $5.4 \%$, es decir 2 estudiantes considera que posee estas capacidades con muy alta presencia, el $40.5 \%$ a saber 15 manifestaron que la presencia de la dimensión es alta, por su parte el 54.1\% equivalente a 20 jóvenes manifiestan que poseen medianamente este aspecto.

Grotberg, en el planteamiento de su teoría expresa que los factores de resiliencia si bien pueden desarrollarse por separado y a consideración del individuo, en el momento enfrentar un episodio adverso deben actuar en con junción. En el caso de estudio se ve reflejado una tendencia medianamente alta para el desarrollo del factor Yo, considerando entonces que los estudiantes de Orientación para el egreso poseen la fuerza interior para salir adelante y conseguir lo que se proponen a futuro respetándose a sí mismo y a los demás asumiendo las consecuencias de sus actos y enfrentando la adversidad con optimismo y lleno de esperanza.

En la siguiente Tabla se muestran los resultados del tercer objetivo que cita: Describir los factores externos de la resiliencia relacionados con el Yo tengo en los estudiantes de Orientación para el egreso, que da respuesta a la dimensión factores externo Yo tengo con sus indicadores apoyo familiar, apoyo social, modelos significativos y entorno social estable.

En el instrumento aplicado se evidencia que la dimensión factores externos por medio del factor Yo tengo, el 21.6\%, es decir 8 estudiantes consideran que el posee apoyo externo con muy alta presencia, el 64.9\%, representado por 24 estudiantes considera que la presencia de esta dimensión es alta, el 13.5\% es decir 5 jóvenes respondieron que la presencia de estos aspectos es media.

En el caso de los factores externos, cabe destacar que el planteamiento del autor hace referencia a que son apoyos externos que permiten la promoción de la resiliencia en el individuo, en el caso de esta investigación se halló que los estudiantes de Orientación para el egreso poseen un alto grado en tener personas a su alrededor que le brindan confianza, aman sin condición marcando límites en su comportamiento, esas personas que los invitan a ser independientes que por lo general son modelos a seguir. En términos generales familia y entorno social estables.

En los resultados se evidencia cómo en la dimensión factores internos desde el factor Yo puedo se ubican de acuerdo al baremo en alta presencia con un porcentaje del $45.9 \%$ con respecto a la aplicación de estas capacidades sociales.

Por su parte en la dimensión factores internos desde el factor Yo soy se ubican con mayor frecuencia en la categoría mediana frecuencia con un porcentaje del $54.1 \%$, sin embargo un número significativo del $40.5 \%$ se ubica con alta frecuencia en la aplicación de estas cualidades relacionadas con la fortalezas internas. 
En cuanto a la dimensión factores externos desde el pilar Yo tengo los resultados reflejan que los estudiantes encuestados se ubican en un $54.1 \%$ en la categoría alta presencia desarrollando relaciones que sirvan de fortaleza para superar las adversidades.

Por lo tanto en cuanto a la variable resiliencia los resultados expuestos reflejan que el $10.4 \%$, es decir 4 estudiantes considera que posee estos pilares con muy alta presencia, el $54.1 \%$ es decir 20 jóvenes manifestaron que la presencia de la variable es alta y por último el $35.1 \%$ a saber 13 encuestados refiere que se posee con mediana presencia resiliencia al momento de enfrentar adversidades, de forma que se puede expresar que poseen alta presencia de resiliencia en su vida pero con un elevado porcentaje de encuestados ubicados en el nivel medio.

La mayoría de las personas cuentan con algunos factores resiliente, y que estos factores pueden a su vez adquirirse a lo largo del crecimiento, sin embargo se necesita el apoyo de otros para el logro de ello. Por su parte la tarea de desarrollar la resiliencia se da con la idea de ladrillos para la construcción del crecimiento comunes de las personas, lo que identifica y delimita los factores de la resiliencia de acuerdo a la edad, por tanto es necesario explorar el desarrollo de los factores de resiliencia según la etapa evolutiva para así considerar su nivel de resiliencia. En términos generales puede decirse que los estudiantes de Orientación para el egreso poseen las capacidades necesarias para superar la adversidad y salir fortalecidos y transformados de ella.

\section{CONCLUSIONES}

A continuación como resultado estadístico de esta investigación se presentan las conclusiones con base a los objetivos formulados para la investigación.

En relación con el primer objetivo: Identificar los factores internos de la resiliencia relacionados con el Yo puedo a través de los indicadores: creatividad, persistencia, buen humor. Asertividad, manejo de conflicto, pedir ayuda se obtuvo que los estudiantes de Orientación para el egreso poseen competencias que le permiten desarrollar este factor de manera óptima, considerando los resultados obtenidos con una alta presencia tendiendo a ser muy alta.

Para dar respuesta al segundo objetivo: Caracterizar los factores internos de la resiliencia relacionados con el Yo soy en los estudiantes de Orientación para el egreso, se consideraron indicadores como: relaciones interpersonales, estabilidad emocional, empatía, metas, respeto y optimismo. Se aprecia que los estudiantes de Orientación para el egreso poseen niveles bajos $y$ medianos en cuanto al desarrollo de capacidades como habilidad emocional y empatía lo que lleva a que a tener como resultado para el factor resiliente $\boldsymbol{Y o}$ soy que poseen medianamente esta capacidad.

Estos resultados guardan correspondencia con los planteamientos de Henderson (2003) y Rutter (1993), los cuales refieren a los factores internos de la resiliencia como una combinación de procesos que permiten a un ser humano afrontar y superar los problemas o adversidades de la vida, así como transformar los eventos en experiencias de aprendizaje. 
De acuerdo al tercer objetivo expresa: Describir los factores externos de la resiliencia relacionados con el Yo tengo en los estudiantes de Orientación para el egreso, tomando en cuenta indicadores como apoyo familiar, apoyo social, modelo significativo, familia estable, los estudiantes de orientación para el egreso poseen relaciones que le permiten el desarrollo de este factor, sin embargo se observó que existe una mediana presencia en cuanto a un entorno social estable y familia estable, lo que indica como resultado del Yo tengo posea una tendencia mediana, cualidades que deben reforzarse.

Sobre este particular, Puig y Rubio (2011); Kent y Davis (2010), Lorenzo (2010); Henderson y Milstein (2003) enfatizan en la importancia de la ayuda familiar, social, comunitaria, organizativa y académica, como factores protectores para fortalecer la conducta prosocial de las personas resilientes, por cuanto pueden modelar significativamente la estabilidad emocional, capacidad de adaptación, disposición hacia los estudios, orientación para evitar o salir fortalecido en situaciones de riesgos o desaciertos

En el desarrollo de la investigación se estima el cuarto objetivo: Establecer la relación entre los factores de la resiliencia presente en estudiantes de Orientación para el egreso considerando una comparación en las dimensiones Factores internos y Factores externos, así como la resiliencia.

De acuerdo a los hallazgos obtenidos, cabe destacar que en los estudiantes de Ingeniería en Petróleo, se subrayan competencias de los factores internos como lo es $\mathbf{Y o}$ puedo y el $\boldsymbol{Y o}$ soy, mientras que presentan cualidades del Yo tengo medias.
En el caso de los estudiantes de Ingeniería Civil, es al contrario, ocurre que sus competencias dentro de los factores internos de resiliencia relacionados con el Yo puedo y Yo soy están medianamente presentes, en tanto a lo que concierne las cualidades resilientes Yo tengo se destacan por su alta presencia.

En definitiva para este objetivo se obtiene que los estudiantes de Ingeniería en Petróleo se encuentren en una categoría alta de resiliencia, mientras que los estudiantes de Ingeniería Civil se encuentran en una categoría media. Lo que hace comprender la gran influencia que representa el factor Yo soy en estos estudiantes.

\section{REFERENCIAS}

Henderson Grotberg, E. (2003). La resiliencia en el mundo de hoy: cómo superar las adversidades. Barcelona: Gedisa

Kent, M. Davis, M. (2010). The Emergence of Capacity-Building Programs and Models of Resilience. En J.W. Reich, A.J. Zautra y J.S. Hall (Eds.), Handbook of adult resilience (pp. 427-449). New York, NY: Guilford

Kenneth, I y Cummings, J (2010). Anchored by faith. Religion as a Resilience Factor. En M. Kent, M. Davis y J. Reich (eds). The Resilience Handbook. Approachesto Stress and Trauma (pp 193-210). New York: Routledge

Lorenzo, R (2010). Resiliencia. Nuestra capacidad de recuperación ante los obstáculos. Editorial Andrómeda, Buenos Aires

Luthar, S. S., Sawyer, J. A., \& Brown, P. J. (2006). Conceptual issues in studies of resilience: Past, present, and future research. Annals of the New York Academy of Sciences, 1094, 105 
Milstein, M., y Henderson, N. (2003). Resiliencia en la Escuela. Buenos Aires: Editorial Paidós

Puig, G. y Rubio, J.L. (2011). Manual de resiliencia aplicada. Barcelona, España:Gedisa

Rutter, M. (1987). Psychosocial resilience and protective mechanisms. American Journal Orthopsychiatry, 57(3), 316-329.

Rutter, M. (1993). Resilience: Some conceptual considerations. Journal of Adolescent Health, 14(8), 626-631
Rutter, M. y Madge, N. (1976). Cycles of disadvantage. Kingston, Gran Bretaña: Heinemann Educational Books

Werner, E. y Smith, R.S. (1982). Vulnerable but invincible: a longitudinal study of resilient children and youth. NuevaYork, E.E.U.U.: McGraw Hill 